\title{
Citri reticulatae Pericarpium attenuates Ang II-induced pathological cardiac hypertrophy via upregulating peroxisome proliferator-activated receptors gamma
}

\author{
Gehui Ni ${ }^{1 \#}$, Kai Wang ${ }^{1 \#}$, Yufei Zhou ${ }^{1}$, Xiaodong Wu ${ }^{1}$, Jiaqi Wang ${ }^{2}$, Hongcai Shang ${ }^{3}$, Lijun Wang ${ }^{2}$, \\ Xinli Li ${ }^{1}$
}

${ }^{1}$ Department of Cardiology, The First Affiliated Hospital of Nanjing Medical University, Nanjing, China; ${ }^{2}$ Cardiac Regeneration and Ageing Lab, Institute of Cardiovascular Sciences, School of Life Science, Shanghai University, Shanghai, China; ${ }^{3}$ Key Laboratory of Chinese Internal Medicine of Ministry of Education, Dongzhimen Hospital, Beijing University of Chinese Medicine, Beijing, China

Contributions: (I) Conception and design: X Li, L Wang, H Shang; (II) Administrative support: X Li, L Wang; (III) Provision of study materials: G Ni, K Wang, Y Zhou, X Wu, J Wang; (IV) Collection and assembly of data: G Ni, K Wang, Y Zhou, X Wu, J Wang; (V) Data analysis and interpretation: G Ni, K Wang, Y Zhou, X Wu, J Wang; (VI) Manuscript writing: All authors; (VII) Final approval of manuscript: All authors.

\#These authors contributed equally to this work.

Correspondence to: Prof. Xinli Li. Department of Cardiology, The First Affiliated Hospital of Nanjing Medical University, 300 Guangzhou Road, Nanjing, China. Email: xinli3267_nj@hotmail.com; Dr. Lijun Wang. Cardiac Regeneration and Ageing Lab, Institute of Cardiovascular Sciences, School of Life Science, Shanghai University, 333 Nan Chen Road, Shanghai, China. Email: lijunwang@shu.edu.cn; Prof. Hongcai Shang. Key Laboratory of Chinese Internal Medicine of Ministry of Education, Dongzhimen Hospital, Beijing University of Chinese Medicine, Beijing, China. Email: shanghongcai@126.com.

Background: Pathological cardiac hypertrophy is a major risk factor for cardiovascular diseases, including heart failure. However, limited pharmacological therapies are available for reversing the maladaptive process and restoring cardiac function. Citri reticulatae Pericarpium (CRP) has been used in traditional Chinese medicine prescriptions for clinical treatment. Previous studies have shown that CRP and its ingredients have beneficial effects on the cardiovascular system. However, whether CRP has a protective effect against pathological cardiac hypertrophy remains unknown.

Methods: Primary neonatal rat cardiomyocytes (NRCMs) were treated with angiotensin II (Ang II) to induce pathological hypertrophy in vitro. Immunofluorescent staining and quantitative real-time PCR (qRT-PCR) were used to determine the cell size and the expression of hypertrophic gene markers (Anp and Bnp), respectively. Male C57BL/6 mice were subjected to the investigation of cardiac hypertrophy induced by Ang II ( $2.5 \mathrm{mg} / \mathrm{kg} / \mathrm{d}$ for 4 weeks). CRP ( $0.5 \mathrm{~g} / \mathrm{kg} / \mathrm{d}$ for 4 weeks) was administrated to treat mice with or without peroxisome proliferatoractivated receptors gamma (PPAR $\gamma$ ) inhibitor T0070907 (1 mg/kg/d for 4 weeks treatment) infused with Ang II. Cardiac hypertrophy (hematoxylin-eosin staining and qRT-PCR), fibrosis (Masson's Trichrome staining, qRTPCR, and western blot), and cardiac function (echocardiography) were examined in these mice. Western blot was used to determine the protein level of PPAR $\gamma$ and PGC- $1 \alpha$ both in NRCMs and in mice.

Results: We found that CRP could prevent Ang II-induced pathological cardiac hypertrophy evidenced by improving cardiac function, decreasing hypertrophic growth and reducing cardiac fibrosis. Also, we demonstrated that PPAR $\gamma$ was upregulated by CRP both in NRCMs and in hearts. Moreover, PPAR $\gamma$ inhibitor could abolish the inhibitory effects of CRP on Ang II-induced pathological cardiac hypertrophy.

Conclusions: CRP attenuates Ang II-induced pathological cardiac hypertrophy by activating PPAR $\gamma$.

Keywords: Pathological cardiac hypertrophy; Citri reticulatae Pericarpium (CRP); peroxisome proliferatoractivated receptors gamma $(\operatorname{PPAR} \gamma)$

Submitted May 02, 2020. Accepted for publication Jul 27, 2020.

doi: 10.21037/atm-20-2118

View this article at: http://dx.doi.org/10.21037/atm-20-2118 


\section{Introduction}

Chronic heart failure is the end-stage of multiple cardiovascular diseases and the leading cause of death (1). Currently, treatment and prevention of heart failure includes physical active lifestyle (e.g., exercise prescription), pharmacological treatment as well as surgical therapy (2-4). Among them, drug therapy is most available due to its better compliance and lower cost in patients. However, the present drug therapy mainly focuses on alleviating symptoms and could not significantly improve the prognosis of patients and reduce the hospitalization rate as well as mortality $(3,4)$. Therefore, identifying new pharmacotherapies and therapeutic approaches for chronic heart failure is highly needed.

Cardiac hypertrophy is defined as cardiomyocyte enlargement in size and ventricular wall thickening in response to an increased workload (5). The compensated heart growth initially is an adaptive response to maintain normal cardiac function, however, sustained stress and changes in the heart become maladaptive, and eventually progress to heart failure. Pathological cardiac hypertrophy, which is accompanied by metabolism and gene expression alterations in cardiomyocytes as well as collagen increase and myofibroblast activation, is a pivotal risk factor for many cardiovascular diseases including heart failure (6). Despite the underlying mechanisms and signaling pathways that have been described in many pathological conditions, limited therapeutic strategies are available for prevention and management of this pathological process $(7,8)$. The peroxisome proliferator-activated receptors gamma $(\operatorname{PPAR} \gamma)$ and its coactivator-1 $\alpha$ (PGC- $1 \alpha)$ play key roles in controlling cardiac metabolism $(9,10)$. PPAR $\gamma$ activation in vivo could ameliorate cardiac hypertrophy and dysfunction (11). Moreover, PPAR $\gamma$ agonists have been widely used in clinical practice because of their important role in metabolic regulation (12). Thus, identifying potential PPAR $\gamma$ modulators is of great clinical value to treat pathological hypertrophy and heart failure.

Citri reticulatae Pericarpium (CRP) (Chenpi in Chinese) is the dried and mature peel of Citrus reticulata Blanco. The cultivars of CRP are mainly distributed in the Guangdong, Guangxi, Sichuan, Fujian, and Zhejiang Provinces of China, belonging to the genus Citrus and family Rutaceae (13). About 140 chemical components have been isolated and identified from CRP, including alkaloids, flavonoids, and essential oils, among which flavonoids are considered to be the primary bioactive constituents of this herbal medicine. CRP has a bitter and acrid flavor, a neutral nature, and is described as possessing the power to regulate qi (13). It has been used in traditional Chinese medicine for thousands of years and was registered in the first edition of the Chinese Pharmacopoeia. In traditional Chinese medicine, CRP has been used for the treatment of diseases pertaining to the digestive and respiratory systems. Moreover, modern pharmacological studies have found that CRP has anti-cancer, antioxidant, anti-inflammatory and other beneficial effects (14-19). Regarding the cardiovascular system, CRP and its ingredients have been reported to improve glucose tolerance and insulin resistance $(20,21)$, to attenuate myocardial ischemia injury and atherosclerosis (22-24). However, whether CRP has a protective effect on pathological cardiac hypertrophy remains unknown.

In the present study, we found that CRP could prevent angiotensin II (Ang II)-induced pathological cardiac hypertrophy via upregulating PPAR $\gamma$. Our data presented here provide experimental evidence for the potential application of CRP in the treatment of pathological cardiac hypertrophy and heart failure. We present the following article in accordance with the ARRIVE reporting checklist (available at http://dx.doi.org/10.21037/atm-20-2118).

\section{Methods}

\section{Animals}

This study was conducted in accordance to the Guidelines for the Care and Use of Laboratory Animals published by the National Institutes of Health (NIH publication, revised in 2011). The study protocol was approved by the ethical animal committees of Nanjing Medical University (license number: IACUC-1903016).

Eight-week-old C57BL/6J male mice were obtained from the Model Animal Research Center of Nanjing University. Each mouse was randomly assigned to each group. CRP was obtained from Shijiazhuang Yiling pharmaceutical CO., LTD (Shijiazhuang, China). Pericarps were isolated and dried under ventilation or low temperatures, and then sprayed, shredded by deionized water twice. The extracting solution was filtered by vibration to remove the impurities. The last extract of CRP was obtained after spray drying. Osmotic mini-pumps (model 2004; Alzet Corp, Palo Alto, CA, USA) were implanted subcutaneously at the dorsum of the neck to infuse a pressor dose of Ang II $(2.5 \mathrm{mg} / \mathrm{kg} / \mathrm{d})$ for 4 weeks. At the same time, mice were administrated with CRP at a dose of $0.5 \mathrm{~g} / \mathrm{kg} / \mathrm{d}$ for 4 weeks via oral gavage. PPAR $\gamma$ inhibitor T0070907 was administrated at a dose of $1 \mathrm{mg} / \mathrm{kg} / \mathrm{d}$ by intraperitoneal injection at the same time with the administration of CRP. 


\section{Echocardiography}

The systolic heart function of the mice was evaluated by echocardiography. Mice were anesthetized with $2 \%$ isoflurane, maintained in the decubitus position and were allowed to breathe spontaneously during the procedure. Echocardiography was performed with Vevo 2100 (Visual Sonics Inc, Toronto, Ontario, Canada). The following parameters were mainly performed: ejection fraction (EF) and left ventricular fractional shortening (FS).

\section{Neonatal rat cardiomyocytes (NRCMs) isolation, culture and treatment}

The primary NRCMs were isolated from 1- to 2-day old Sprague Dawley rats. Heart tissues were collected as previously described (25). Briefly, heart tissues were digested, and purified by Percoll gradient centrifugation. Subsequently, the NRCMs were re-suspended in high glucose Dulbecco's modified Eagle's medium (DMEM) (Gibco, Pasadena, CA, USA) with 5\% fetal bovine serum (FBS) (Gibco, Pasadena, CA, USA), 10\% horse serum (HS) (Gibco, Pasadena, CA, USA), 1\% penicillin-streptomycin and then plated to appropriate culture dishes. To induce Ang II-induced cardiomyocytes hypertrophy, NRCMs were firstly starved for 8 hours in serum-free DMEM and exposed to $1 \mu \mathrm{M}$ AngII (Sigma, St. Louis, MO, USA) for 48 hours. To investigate the roles of CRP in AngII-induced NRCM hypertrophy, NRCMs were treated simultaneously with CRP $(0.5 \mu \mathrm{g} / \mathrm{mL})$ (Shijiazhuang Yiling Pharmaceutical Co., Hebei, China) for 48 hours. To investigate whether CRP protected NRCMs from cardiac hypertrophy via PPAR $\gamma$, NRCMs were treated simultaneously with CRP together with $1 \mu \mathrm{M}$ PPAR $\gamma$ agonist (Rosiglitazone) or $1 \mu \mathrm{M}$ PPAR $\gamma$ inhibitor (T0070907) as well as Ang II for 48 hours.

\section{Hematoxylin-eosin (H\&E) staining}

Heart tissues were fixed with $4 \%$ paraformaldehyde, and the H\&E staining was performed on the paraffin sections. Nikon Eclipse microscope with NIS Elements software was used to capture the images. Image J software was used to evaluate the cross-sectional area.

\section{Masson's trichrome staining}

Heart tissues were fixed in $4 \%$ paraformaldehyde, embedded in paraffin. $5 \mu \mathrm{m}$ thick sections were subjected to Masson's trichrome staining. Images were captured by a Nikon Eclipse microscope with NIS Elements software and the fibrotic area was dyed blue. Image-Pro Plus software was used to measure the fibrotic area. The fibrotic fraction was calculated as a percentage of the fibrotic area to the total myocardial area.

\section{Western blot}

The NRCMs or cardiac tissues were lysed using RIPA buffer (Beyotime, Nantong, China) with $1 \mathrm{mM}$ PMSF (ST505, Beyondtime. Nantong, China), and the concentration of protein samples was evaluated by BCA protein assay kit (Beyotime, Nantong, China). Equal amounts of protein were separated in 10\% SDS-PAGE and transferred into PVDF membranes which incubated with $5 \%$ milk and with primary antibody at $4{ }^{\circ} \mathrm{C}$ overnight. The primary antibodies were used as follow: PPAR $\gamma(1: 1,000$, Proteintech Group, 16643-1-AP, Wuhan, China), PGC-1 $\alpha$ (1:1,000, Novus Biologicals, NBP2-37562, Littleton, COLO, USA), Collagen I (1:1,000, Wanleibio, WL0088, Shenyang, China), $\alpha$-SMA (1:4,000, Proteintech Group, 23081-1-AP ,Wuhan, China), GAPDH (1:1,000, Kangchen, KC-5G4, Shanghai, China). The next day, the membranes were incubated with the secondary antibodies and signals were visualized using the ECL Plus Western blotting detection reagents (Bio-Rad, Hercules, CA, USA) and the ChemiDoc XRS Plus luminescent image analyzer (BioRad, Hercules, CA, USA). Densitometric analysis of band intensity was performed using Imagelab software (Bio-Rad, Hercules, CA, USA). GAPDH was used as loading controls for total protein expression.

\section{Quantitative real-time PCR (qRT-PCR)}

The NRCMs or heart tissues were lysed using TRIZOL RNA extraction kit (Invitrogen, USA) and reverse transcribed to cDNAs using iScript ${ }^{\mathrm{TM}}$ cDNA Synthesis Kit (Bio-Rad, USA) according to the manufacturer's instructions. Real-Time PCRs were used to determine gene expression levels by using an ABI 7900HT fast RealTime PCR System (Applied Biosystems) with SYBR-Green supermix Kit (Bio-Rad). Gapdh was used as an internal control. The primer sequences used in this study are listed as follows:

Anp (rat): F: 5'-GAGCAAATCCCGTATACAGTGC-3', R: 5'-ATCTTCTACCGGCATCTTCTCC-3'.

$B n p$ (rat): F: 5'-GCTGCTGGAGCTGATAAGAGAA-3', 


\section{R: 5'-GTTCTTTTGTAGGGCCTTGGTC-3'. Gapdh (rat): F: 5'-TGACAACTCCCTCAAGATTGTCA-3', R: 5'-GGCATGGACTGTGGTCATGA-3'. \\ Anp (mouse): F: 5'-AGGCAGTCGATTCTGCTT-3', R: 5'-CGTGATAGATGAAGGCAGGAAG-3'. \\ Bnp (mouse): F: 5'-TAGCCAGTCTCCAGAGCAATTC-3', R: 5'-TTGGTCCTTCAAGAGCTGTCTC-3'. \\ Gapdh (mouse): F: 5'-AGGTCGGTGTGAACGGATTTG-3', R: 5'-TGTAGACCATGTAGTTGAGGTCA-3'. \\ $\alpha-S M A$ (mouse): F: 5'-GTCCCAGACATCAGGGAGTAA-3', R: 5'-TCGGATACTTCAGCGTCAGGA-3'. \\ Colla1 (mouse): F: 5'-GAGCGGAGAGTACTGGATCGA-3', R: 5'-CTGACCTGTCTCCATGTTGCA-3'. \\ Col3a1 (mouse): F: 5'-CTGTAACATGGAAACTGGGGAAA-3', R: 5'-CCATGACTGAACTGAAAACCACC-3'.}

\section{Immunofluorescent staining for $\alpha$-actinin and cell size determination}

The NRCMs were fixed in $4 \%$ paraformaldehyde and permeabilized with $0.2 \%$ Triton $\mathrm{X}-100$ each for 20 minutes at room temperature. Then NRCMs were blocked with $10 \%$ goat serum in PBS for 1 hour at room temperature and incubated with primary antibody $\alpha$-actinin overnight (1:200) (Sigma-Aldrich, St, MO, USA). Nuclei were stained with DAPI (Sigma-Aldrich, St, MO, USA). The images were captured by the inverted fluorescent microscope (Carl Zeiss) and the cell surface area was analyzed using software ImageJ Launcher.

\section{Statistical analysis}

All data were presented as mean \pm standard deviation (SD). The statistical significance was assessed using oneway ANOVA for multiple-group comparison followed by Bonferroni's post hoc test. $\mathrm{P}$ value less than 0.05 was considered to be statistically significant. All data were analyzed by GraphPad Prism 7 software.

\section{Results}

\section{CRP alleviates Ang II-induced hypertrophy in cardiomyocytes}

To investigate the role of CRP in the heart, we first assessed the effect of CRP on pathological hypertrophy induced by Ang II in cultured NRCMs. As shown in Figure $1 A$, immunostaining of NRCMs for $\alpha$-actinin indicated that CRP treatment of NRCMs attenuated the Ang II-stimulated increase in cardiomyocyte size. Next, we examined the mRNA levels of natriuretic peptide type $\mathrm{A}(A n p)$ and natriuretic peptide $\mathrm{B}(B n p)$, and found that CRP-treatment significantly prevented the Ang II-induced hypertrophic marker ( $A n p$ and Bnp) expression in NRCMs (Figure 1B). Taken together, these data suggested that CRP ameliorates Ang II-induced NRCMs hypertrophy.

\section{CRP attenuates cardiac bypertrophy and cardiac fibrosis induced by Ang II infusion in vivo}

To further test whether CRP contributes to the amelioration of Ang II-induced cardiac hypertrophy and remodeling in vivo, wild type (WT) mice with and without CRP treatment were subjected to subcutaneously infusion of Ang II for 4 weeks. As demonstrated by echocardiographs in Figure 2A, Ang II infusion resulted in significant decrease in the ejection fraction (EF) and fractional shortening (FS) compared with saline controls, while CRP treatment prevented Ang II-induced cardiac dysfunction. Histological analysis with $\mathrm{H} \& \mathrm{E}$ staining revealed that the increased cellular cross-sectional area induced by Ang II was dramatically attenuated in CRP treatment mice (Figure 2B). CRP could also alleviate the upregulation of hypertrophic markers (Anp and Bnp) mRNA levels induced by Ang II infusion (Figure 2C). Next, the ameliorated cardiac fibrosis was also characterized by reduction of fibrotic areas (Figure $3 A$ ). To further confirm the anti-fibrotic effect of CRP, we detected the fibrosis-related genes $(\alpha-S M A$, Col1a1, Col3a1) mRNA level by qRT-PCR, and found that treatment with CRP attenuated the Ang II-induced upregulation of fibrotic genes (Figure 3B). Consistently, the protein level of $\alpha$-SMA and collagen I were dramatically downregulated in CRP-treated mice after Ang II infusion (Figure 3C). Collectively, our data suggested that CRP could improve cardiac function induced by Ang II infusion via ameliorating the pathological cardiac hypertrophy and cardiac fibrosis.

\section{PPARy and PGC-1 $\alpha$ are upregulated by CRP both in NRCMs and in mice bearts}

The alterations in cardiac metabolic substrates during cardiovascular diseases have been reported previously (26-28). PPAR $\gamma / \mathrm{PGC}-1 \alpha$ are key regulators in cardiac metabolism, then we examined the expression of PPAR $\gamma$ and its coactivator PGC- $1 \alpha$ in response to CRP treatment. 
A $\alpha$-actinin/DAPI
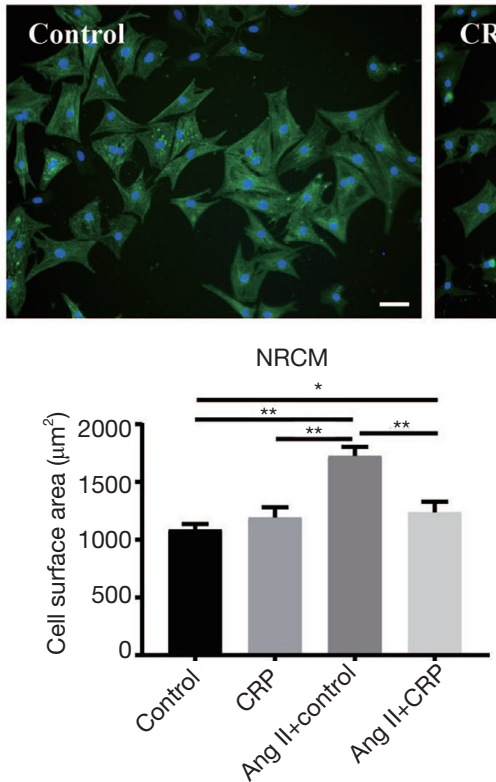
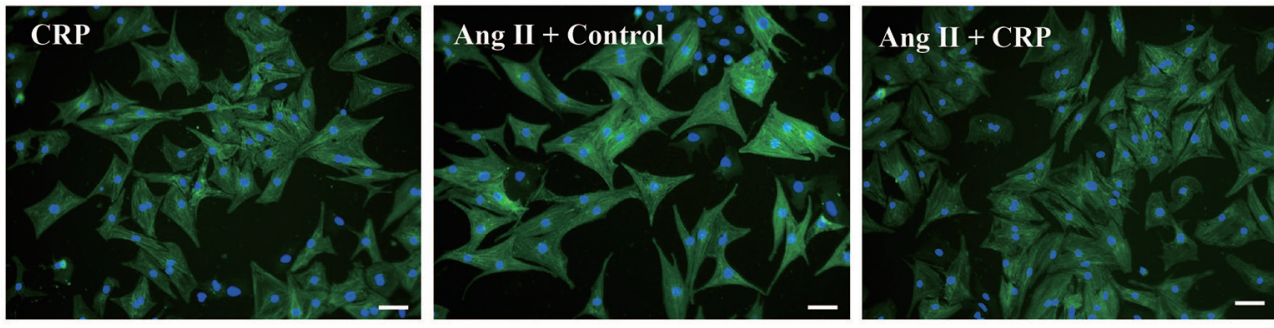

B
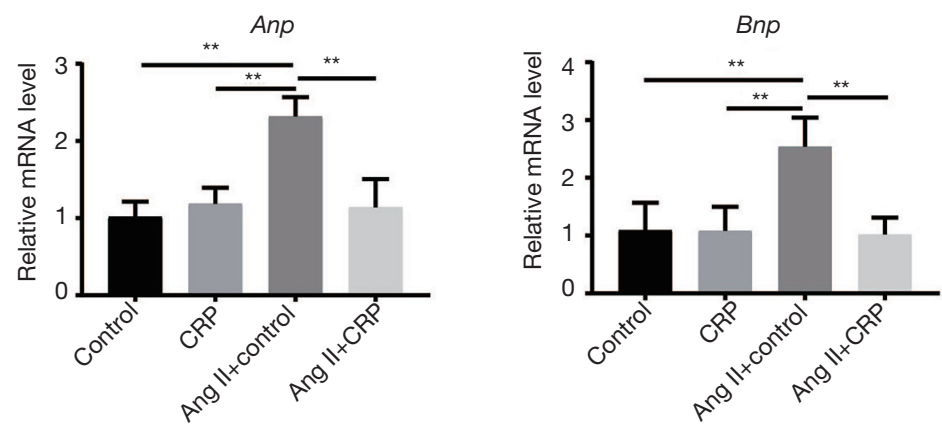

Figure 1 CRP alleviates Ang II-induced hypertrophy in cardiomyocytes. (A) Immunostaining of NRCMs for $\alpha$-actinin indicated that CRP treatment of NRCMs attenuated the Ang II-stimulated increase in cardiomyocyte size, scale bars =50 $\mu \mathrm{m}(\mathrm{n}=6)$; (B) qRT-PCR results showed that CRP-treatment significantly prevented the Ang II-induced hypertrophic marker (Anp and Bnp) expression in NRCMs ( $=6$ ). Data are presented as mean $\pm \mathrm{SD}$. *, $\mathrm{P}<0.05 ;{ }^{* *}, \mathrm{P}<0.01$. CRP, Citri reticulatae Pericarpium; Ang II, angiotensin II; NRCMs, neonatal rat cardiomyocytes; Anp, natriuretic peptide type A; Bnp, natriuretic peptide B.

We found that the expression of PPAR $\gamma$ and its coactivator PGC-1 $\alpha$ declined significantly in Ang II-induced NRCM hypertrophy, while the expression of PPAR $\gamma$ and PGC$1 \alpha$ in CRP treatment group was up-regulated (Figure $4 A$ ). Next, we asked whether those regulation effects observed in vivo, western blot was used to detect the expression of PPAR $\gamma$ and PGC- $1 \alpha$ in the mice heart tissues of cardiac hypertrophy stimulated by Ang II. As shown in Figure 4B, CRP increased PPAR $\gamma$ and PGC- $1 \alpha$ expression in vivo. These results suggested that PPAR $\gamma$ and PGC- $1 \alpha$ are upregulated by CRP both in NRCMs and in mice hearts.

\section{CRP alleviates Ang II-induced cardiomyocytes bypertrophy via PPARy}

Next, we analyzed whether CRP-mediated PPAR $\gamma$ activation contributes to the protective effect of CRP on NRCM hypertrophy stimulated by Ang II. As demonstrated by immunostaining of NRCMs for $\alpha$-actinin and qRTPCR detection for the expression of hypertrophic genes (Anp and Bnp), PPAR $\gamma$ inhibitor T0070907 $(1 \mu M)$ abolished the preventive effects of CRP in Ang II- stimulated cardiomyocyte hypertrophy, whereas PPAR $\gamma$ agonist Rosiglitazone $(1 \mu \mathrm{M})$ did not exert additional protective effect (Figure 4C,D). Taken together, these results demonstrated that CRP attenuates Ang II-induced hypertrophy in NRCMs via maintaining the activation of PPAR $\gamma$.

\section{CRP protects from Ang II-induced cardiac dysfunction via $P P A R \gamma$}

To further determine whether the activation of PPAR $\gamma$ is a critical downstream effector of the CRP beneficial effects in Ang II-induced cardiac dysfunction, PPAR $\gamma$ inhibitor $(1 \mathrm{mg} / \mathrm{kg} / \mathrm{d})$ was treated together with CRP on mice. As shown in Figure 5A, PPAR $\gamma$ inhibitor abolished the prevention of CRP on Ang II-induced cardiac dysfunction evidenced by echocardiography (EF and FS). Besides, PPAR $\gamma$ inhibitor treatment blocked CRP-mediated inhibition of Ang IIinduced pathological hypertrophy phenotypes, including enlargement of cell size and expression of hypertrophic genes (Anp and Bnp) (Figure 5B,C). Moreover, the anti-fibrotic effect of CRP on Ang II infusion hearts was blunted by 


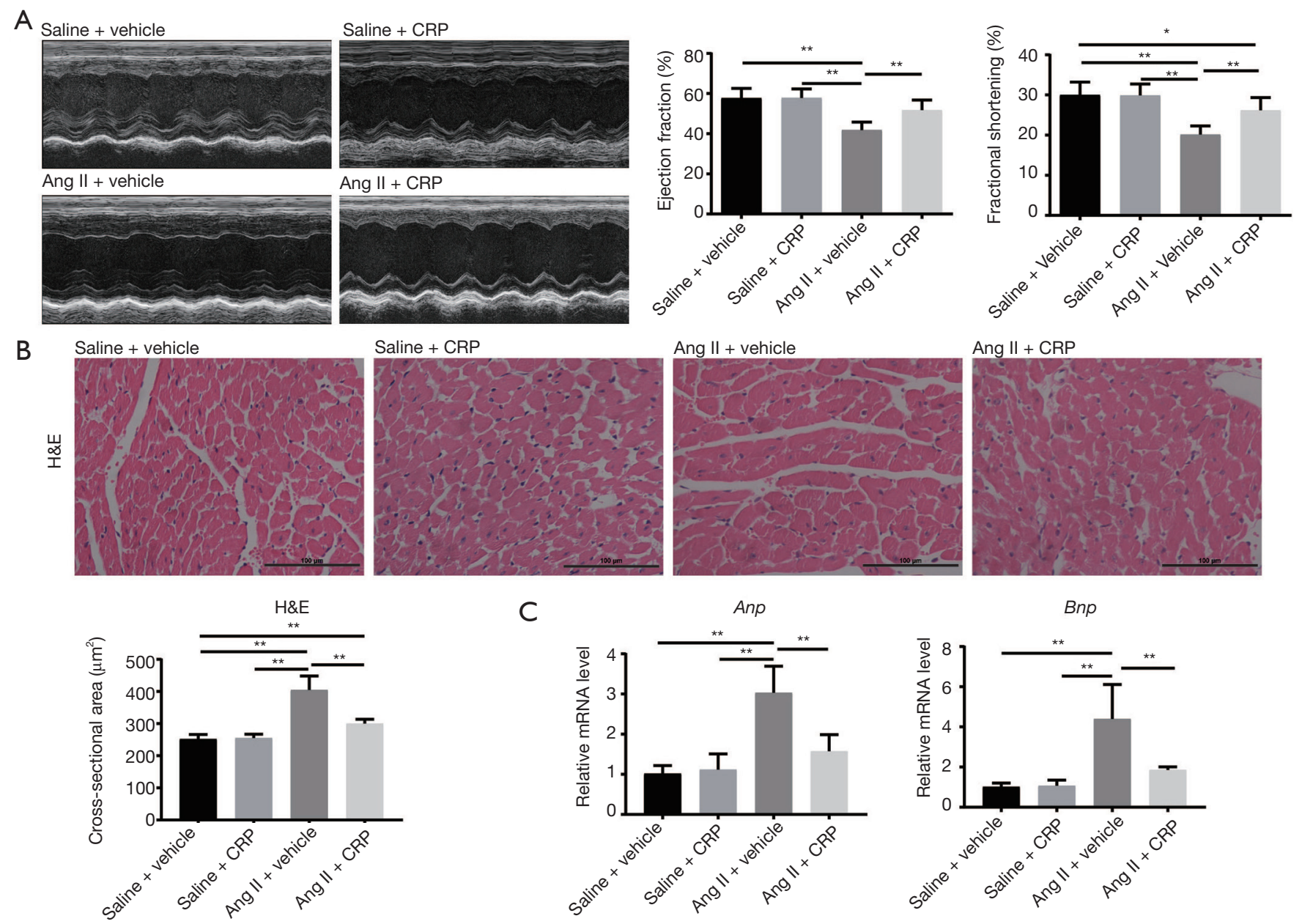

Figure 2 CRP improves cardiac function and attenuates cardiac hypertrophy induced by Ang II infusion in vivo. (A) Echocardiography for mice indicated that CRP treatment prevented Ang II-induced cardiac dysfunction with recovery of the ejection fraction (EF) and fractional shortening (FS) (n=8, 8, 9, 10); (B) histological analysis with haematoxylin and eosin (H\&E) staining revealed that the increased cross-sectional area induced by Ang II was dramatically attenuated in CRP treatment mice, scale bars $=100 \mu \mathrm{m}$ (n=8, 8, 9, 9); (C) qRT-PCR results showed that CRP alleviated the upregulation of hypertrophic markers (Anp and Bnp) expression induced by Ang II infusion in mice ( $\mathrm{n}=8,8,9$, 10). Data are presented as mean $\pm \mathrm{SD}$. *, $\mathrm{P}<0.05$; ** $\mathrm{P}<0.01$. CRP, Citri reticulatae Pericarpium; Ang II, angiotensin II; NRCMs, neonatal rat cardiomyocytes; Anp, natriuretic peptide type A; Bnp, natriuretic peptide B.

PPAR $\gamma$ inhibitor as evidenced by histological analysis with Masson-Trichrome staining (Figure 6A), mRNA levels of fibrotic genes ( $\alpha-S M A$, Colla1, and Col3a1) by qRT-PCR (Figure $6 B$ ), and protein levels of $\alpha$-SMA and collagen I (Figure 6C). These results indicated that activation of PPAR $\gamma$ is essential for the protective effect of CRP on Ang II-induced cardiac hypertrophy and fibrosis.

\section{Discussion}

Pathological cardiac hypertrophy is a major risk factor for cardiovascular diseases, including heart failure. However, limited pharmacological therapies are available for reversing the maladaptive process and restoring cardiac function. Seeking for new therapies and strategies represents a promising therapeutic prospect. CRP, as a typical Chinese herbal medicine, has been used in traditional Chinese medicine prescriptions for clinical treatment $(29,30)$. In the present study, we demonstrated that CRP protected against pathological cardiac hypertrophy induced by Ang II stimulation in NRCMs. In vivo, CRP attenuated Ang II-induced cardiac hypertrophy and fibrosis and rescued 
A

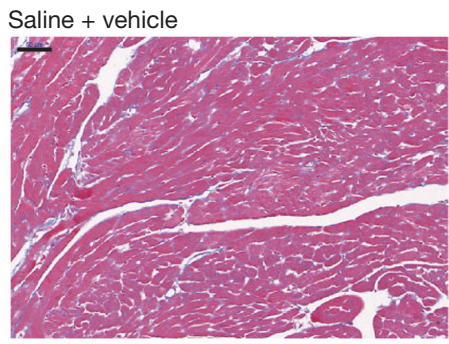

Ang II + vehicle

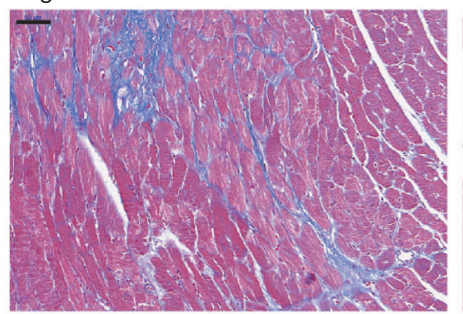

B

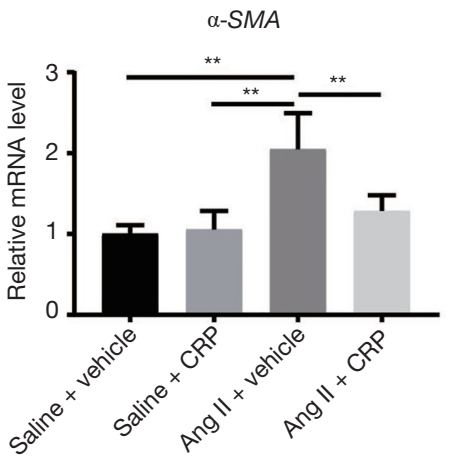

Saline + CRP

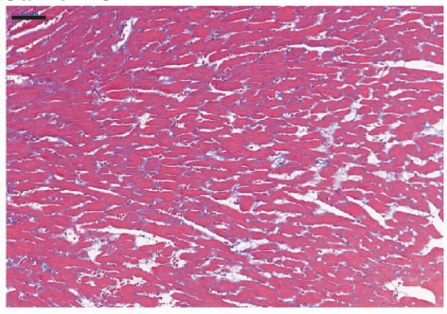

Ang II + CRP

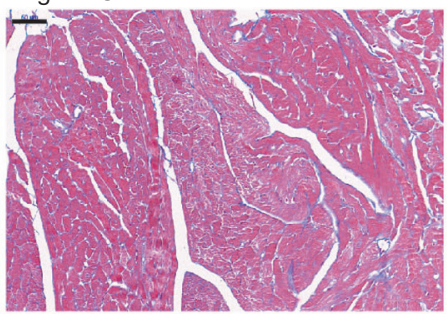

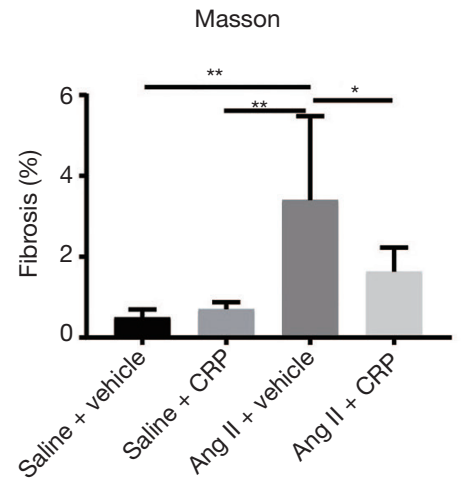

C

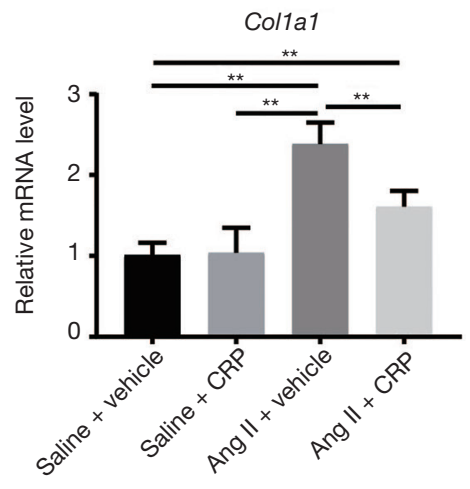

Saline + vehicle Saline + CRP Ang II + vehicle Ang II + CRP
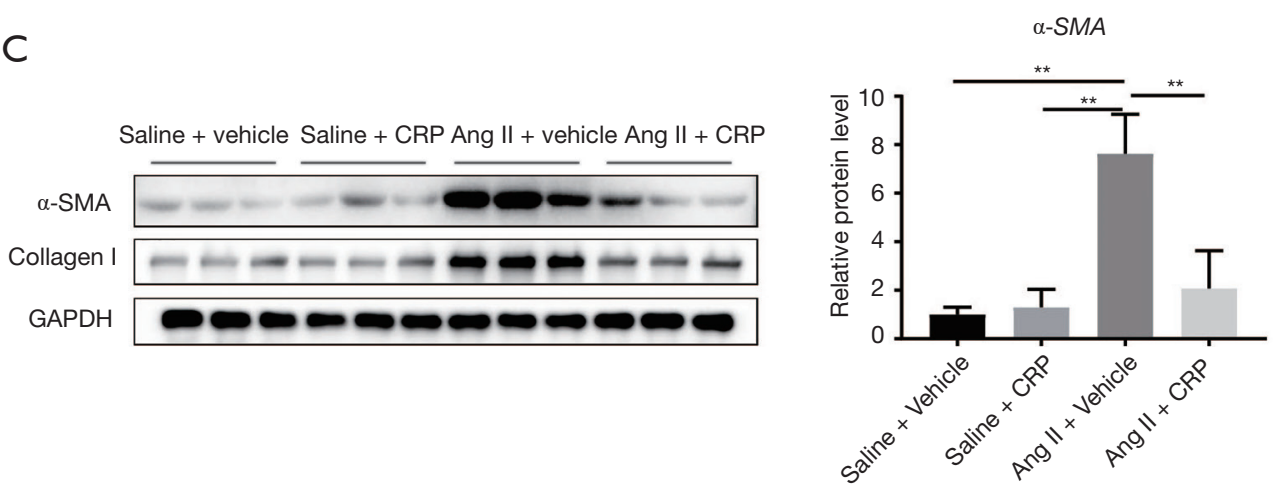

Collagen I

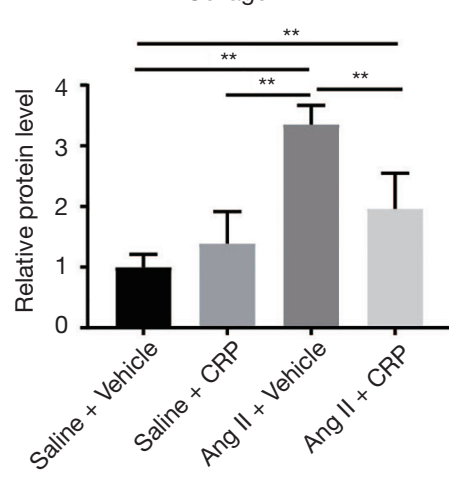

Figure 3 CRP alleviates cardiac fibrosis induced by Ang II infusion in vivo. (A) Masson's trichrome staining revealed that CRP decreased cardiac fibrosis in Ang II infusion in mice, scale bars $=50 \mu \mathrm{m}(\mathrm{n}=8,8,9$, 9); (B) qRT-PCR analysis of $\alpha$-SMA, Col1a1 and Col3a1 expression showed that CRP treatment attenuated the Ang II-induced upregulation of above fibrotic genes in mice ( $\mathrm{n}=8,8,9,10)$; (C) Western blot analysis of treatment with or without CRP indicated that the protein level of Collagen I and $\alpha$-SMA were dramatically downregulated in CRP-treated mice after Ang II infusion ( $\mathrm{n}=6$ ). Data are presented as mean \pm SD. *, $\mathrm{P}<0.05$; ${ }^{*}, \mathrm{P}<0.01$. CRP, Citri reticulatae Pericarpium; Ang II, angiotensin II. 
A

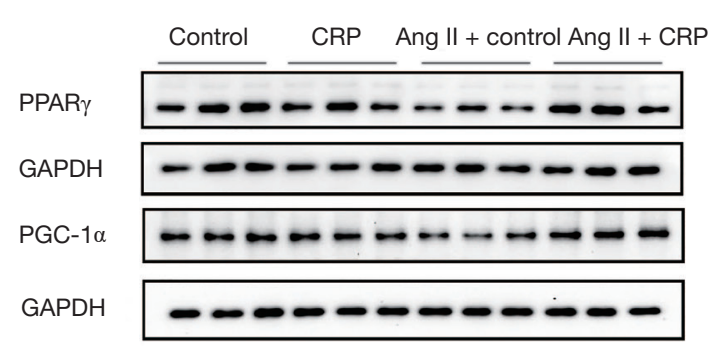

B

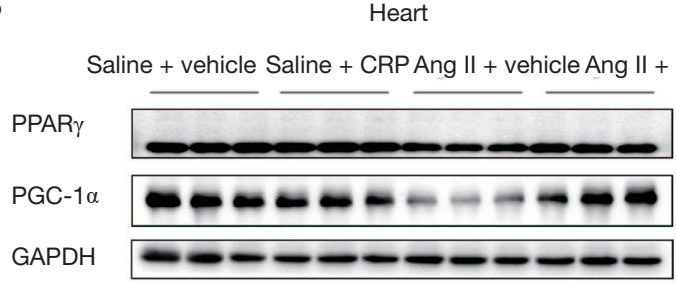

C
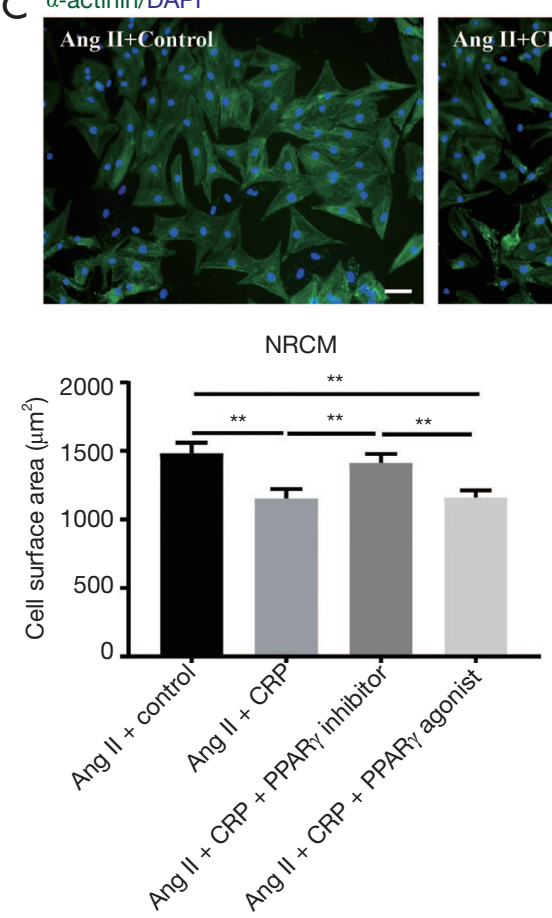

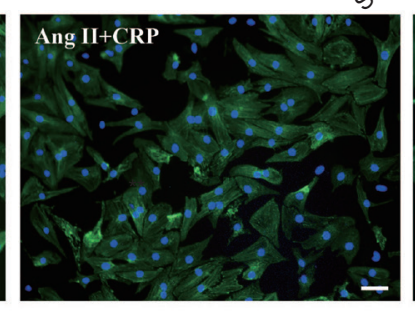

D

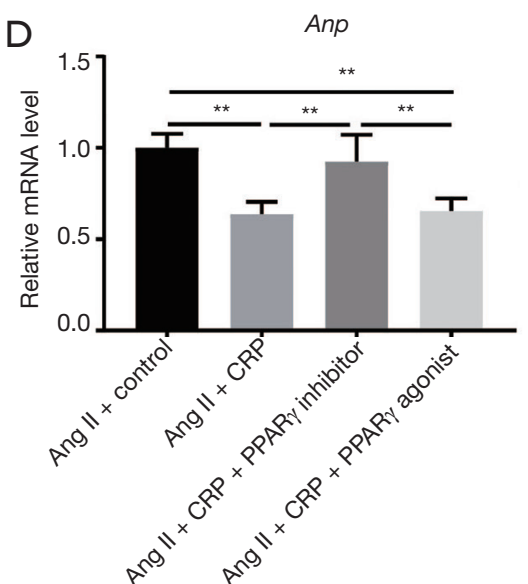

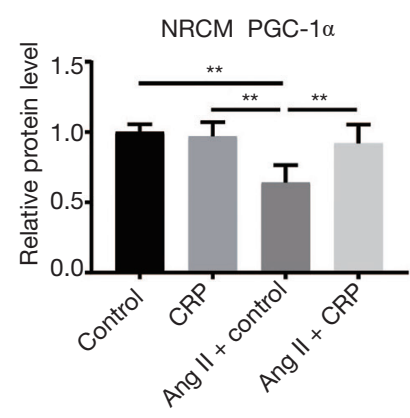

Heart PGC-1 $\alpha$
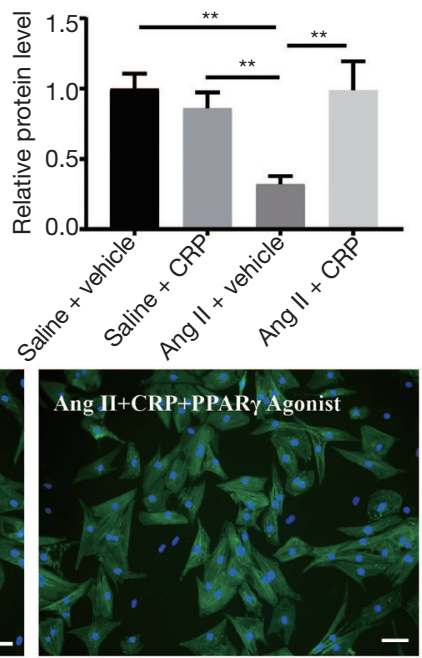

Bnp

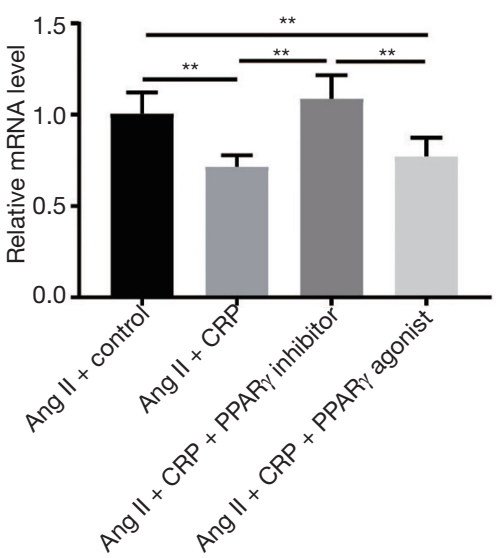

Figure 4 CRP alleviates Ang II-induced hypertrophy via PPAR $\gamma$. (A,B) Western blot analysis of treatment with or without CRP revealed that PPAR $\gamma$ and PGC-1 $\alpha$ were positively regulated by CRP both in NRCMs and in mice hearts (n=6); (C) immunofluorescent staining of NRCMs showed that PPAR $\gamma$ inhibitor T0070907 $(1 \mu \mathrm{M})$ abolished the preventing effects of CRP in Ang II-stimulated cardiomyocyte hypertrophy, whereas PPAR $\gamma$ agonist Rosiglitazone $(1 \mu \mathrm{M})$ did not exert additional protective effect, scale bars $=50 \mu \mathrm{m}(\mathrm{n}=6)$; $(\mathrm{D}) \mathrm{qRT}$-PCR analysis of hypertrophic marker (Anp and Bnp) expression indicated that PPAR $\gamma$ inhibitor T0070907 (1 $\mu \mathrm{M})$ blocked the protective effects of CRP in Ang II-stimulated cardiomyocyte hypertrophy, though PPAR $\gamma$ agonist Rosiglitazone $(1 \mu M)$ did not further enhance the preventing effects of CRP $(\mathrm{n}=6)$. Data are presented as mean $\pm \mathrm{SD}$. ${ }^{*}, \mathrm{P}<0.05 ;{ }^{* *}, \mathrm{P}<0.01$. CRP, Citri reticulatae Pericarpium; Ang II, angiotensin II; NRCMs, neonatal rat cardiomyocytes; Anp, natriuretic peptide type A; Bnp, natriuretic peptide B; PPAR $\gamma$, peroxisome proliferator-activated receptor gamma; PGC- $1 \alpha$, PPAR $\gamma$ coactivator- $1 \alpha$. 
A

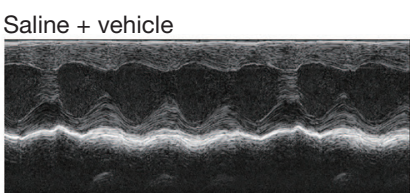

Ang II + CRP

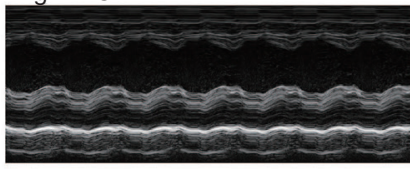

B
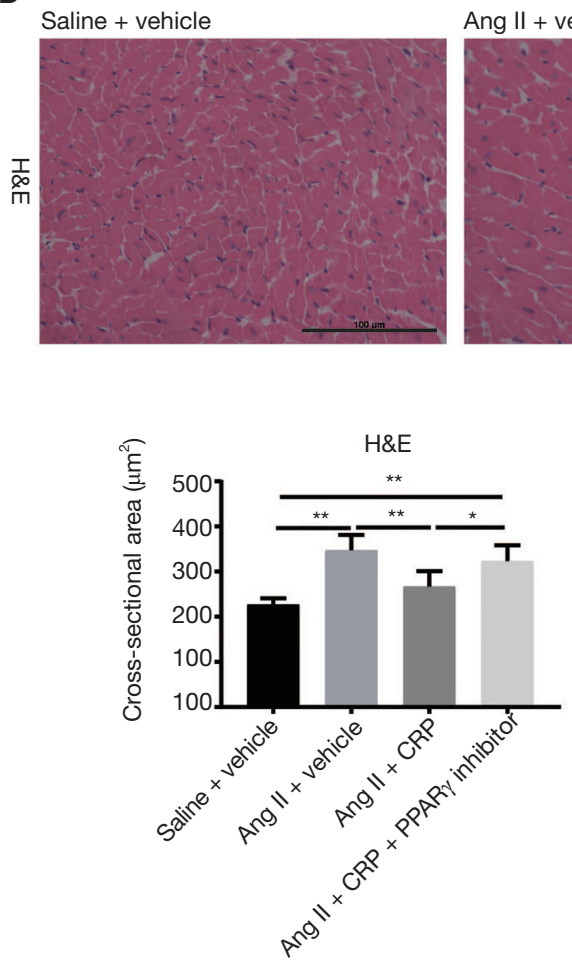

Ang II + vehicle

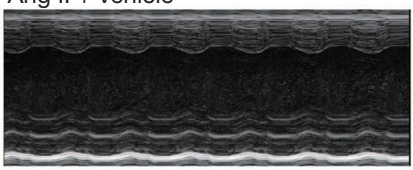

Ang II + CRP + PPAR $\gamma$ inhibitor

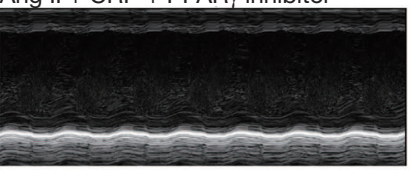

Ang II + vehicle

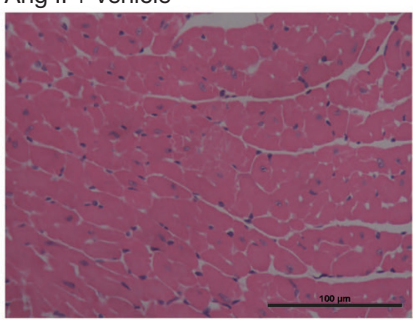

C

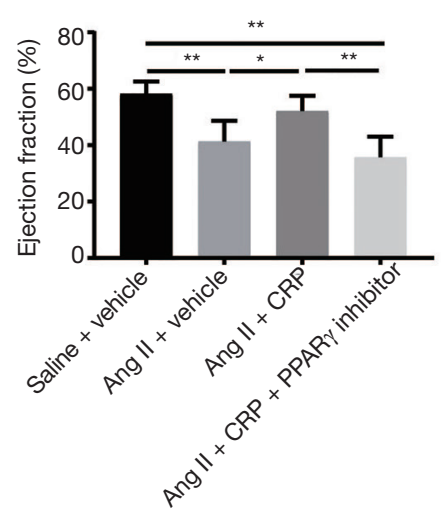

Ang II + CRP
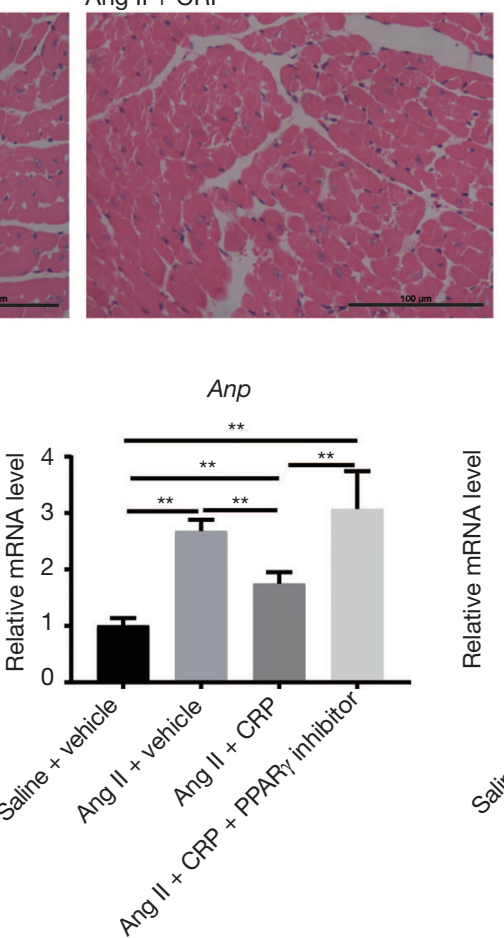

Ang II + CRP + PPAR $\gamma$ inhibitor

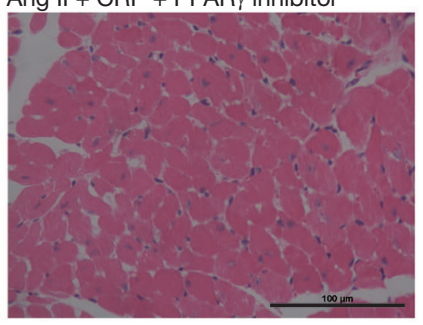

Bnp
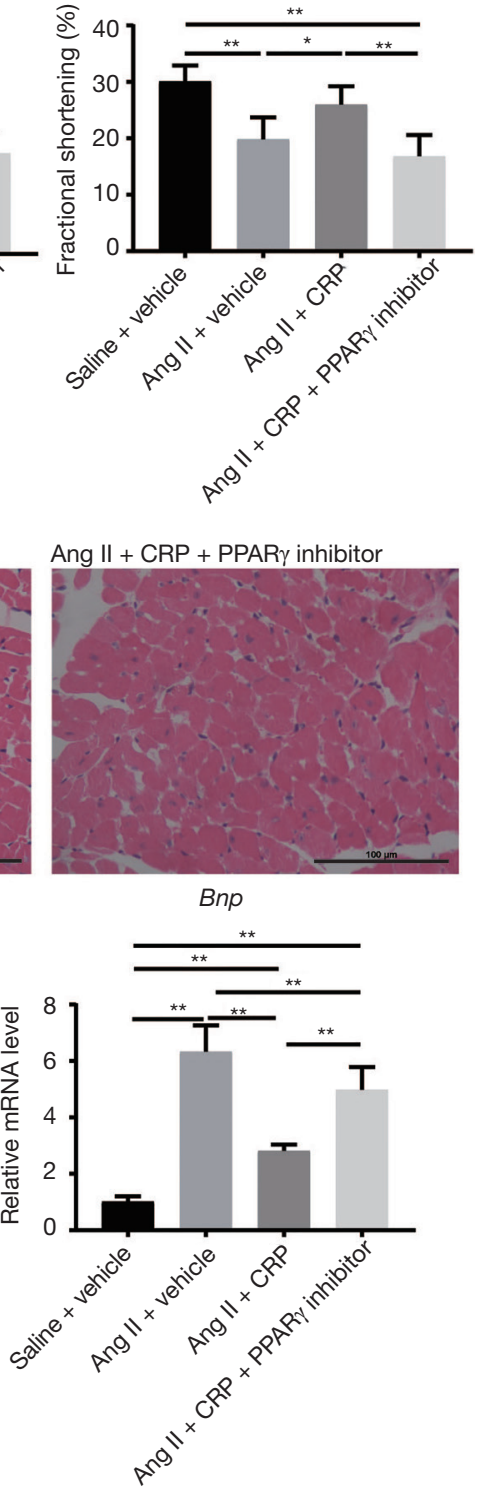

Figure 5 CRP increases cardiac function and ameliorates cardiac hypertrophy via PPAR $\gamma$. (A) PPAR $\gamma$ inhibitor (1 mg/kg/d) abolished the prevention of CRP on Ang II-induced cardiac dysfunction evidenced by echocardiography for mice including decrease of ejection fraction (EF) and fractional shortening (FS) (n=8, 6, 6, 6); (B) Histological analysis with haematoxylin and eosin (H\&E) staining revealed that PPAR $\gamma$ inhibitor $(1 \mathrm{mg} / \mathrm{kg} / \mathrm{d})$ blocked the CRP-mediated inhibition of Ang II-induced pathological hypertrophy proved by enlargement of cell size in mice, scale bars $=100 \mu \mathrm{m}(\mathrm{n}=8,6,6,6)$; (C) qRT-PCR results showed that PPAR $\gamma$ inhibitor $(1 \mathrm{mg} / \mathrm{kg} / \mathrm{d}) \mathrm{blocked} \mathrm{the} \mathrm{prevention} \mathrm{of} \mathrm{CRP}$ on Ang II-induced pathological hypertrophy evidenced by upregulation of hypertrophic markers (Anp and Bnp) expression in mice ( $\mathrm{n}=8$, 6, 6, 6). Data are presented as mean \pm SD. *, $\mathrm{P}<0.05$; **, $\mathrm{P}<0.01$.CRP, Citri reticulatae Pericarpium; Ang II, angiotensin II; NRCMs, neonatal rat cardiomyocytes; Anp, natriuretic peptide type A; Bnp, natriuretic peptide B; PPAR $\gamma$, peroxisome proliferator-activated receptor gamma. 
A

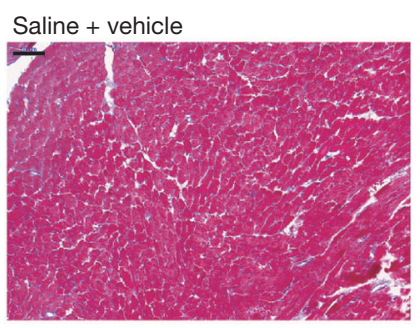

Ang II + CRP

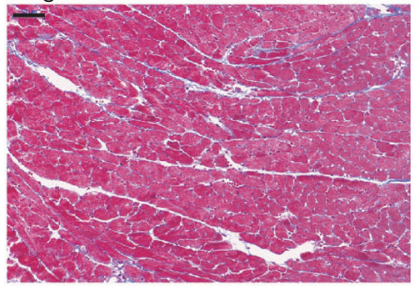

B

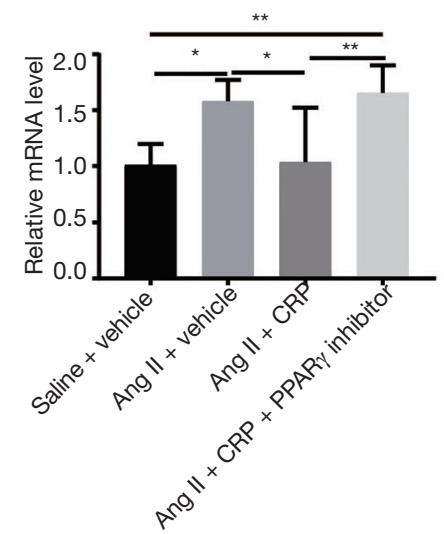

Ang II + vehicle

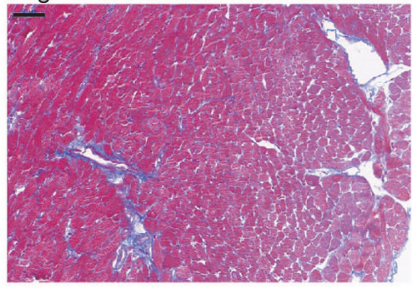

Ang II + CRP + PPAR $\gamma$ inhibitor

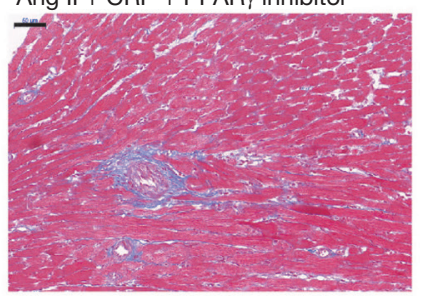

Col1a1

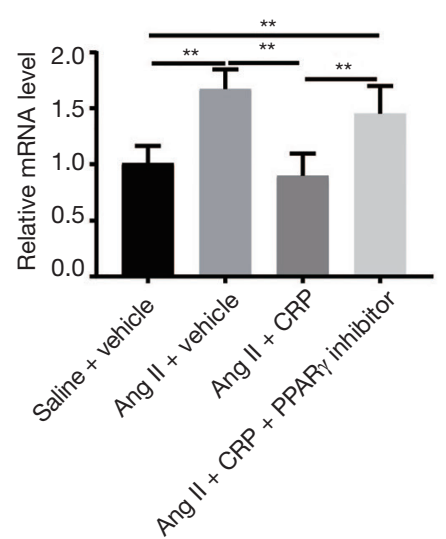

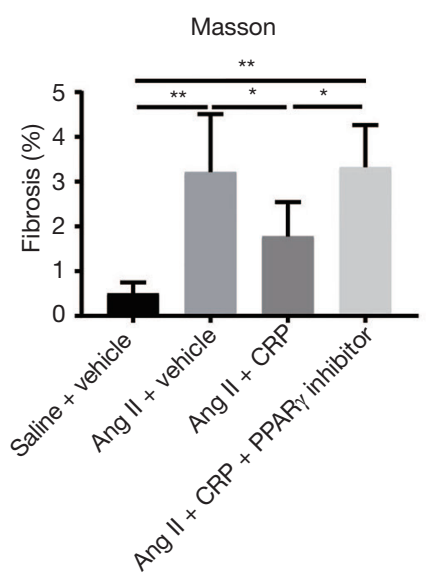

Col3a1

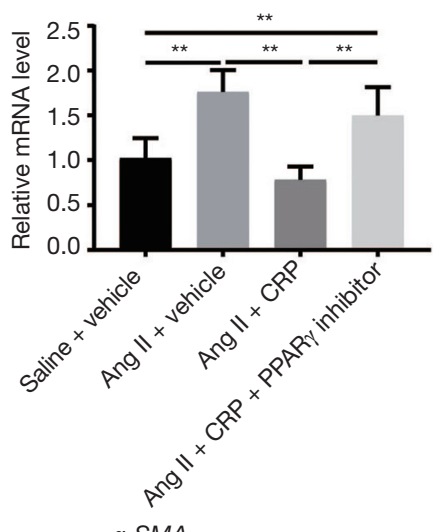

Collagen I

C

Saline + vehicle Ang II + vehicle Ang II + CRP Ang II + CRP + PPAR $\gamma$ inhibitor $\bar{\Phi}$

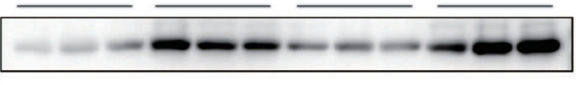

Collagen I

$--\infty-\infty-\infty-\infty \omega$

GAPDH

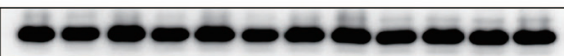

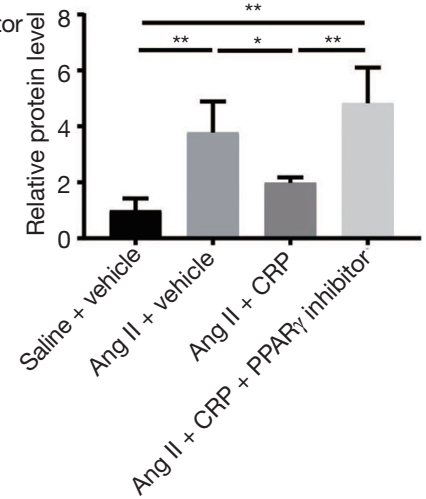

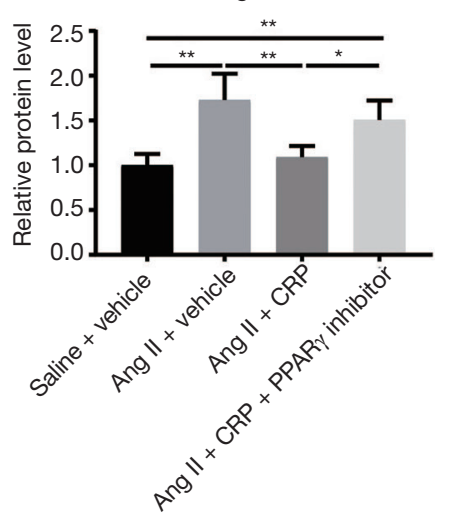

Figure $6 \mathrm{CRP}$ attenuates cardiac fibrosis induced by Ang II infusion via PPAR $\gamma$ in vivo. (A) Masson's trichrome staining revealed that PPAR $\gamma$ inhibitor $(1 \mathrm{mg} / \mathrm{kg} / \mathrm{d}$ ) blunted the anti-fibrotic effect of CRP in Ang II infusion hearts, scale bars =50 $\mu \mathrm{m}$ (n=8, 6, 6, 6); (B) qRTPCR analysis of $\alpha-S M A$, Colla1 and Col3a1 expression showed that PPAR $\gamma$ inhibitor $(1 \mathrm{mg} / \mathrm{kg} / \mathrm{d})$ abolished the protective effect of CRP on cardiac fibrosis induced by Ang II infusion ( $\mathrm{n}=8,6,6,6)$; (C) the results of western blot indicated that the protein level of collagen I and $\alpha$-SMA were dramatically upregulated in PPAR $\gamma$ inhibitor-treated mice $(1 \mathrm{mg} / \mathrm{kg} / \mathrm{d})(\mathrm{n}=6)$. Data are presented as mean $\pm \mathrm{SD}$. *, $\mathrm{P}<0.05 ;$ **, $\mathrm{P}<0.01$. CRP, Citri reticulatae Pericarpium; Ang II, angiotensin II; PPAR $\gamma$, peroxisome proliferator-activated receptor gamma. 
cardiac function. PPAR $\gamma$ and PGC-1 $\alpha$ were upregulated by CRP. Further functional rescue study showed that PPAR $\gamma$ inhibitor blocked the cardioprotective effects of CRP both in NRCMs and in vivo.

Cardiac hypertrophy is characterized by cardiomyocyte enlargement, ventricular wall thickening, and shift in fetal genes expression. During this process of cardiac compensatory growth, metabolism impairment often occurs in cardiomyocytes. PPAR $\gamma$ and its coactivators PGC- $1 \alpha$ are well-known regulators involved in regulating cardiac energetic homeostasis (31). PPAR $\gamma$ is a ligand-activated transcription factor belonging to the nuclear receptor family of peroxisomal proliferator activated receptor (PPAR), which is highly expressed in adipocytes, vascular smooth muscle cells, macrophages, cardiomyocytes, and endothelial cells. Previous studies have found that activation of PPAR $\gamma$ could improve myocardial ischemia/ reperfusion injury (32), alleviate myocardial fibrosis (33), reduce myocardial apoptosis (34), inhibit myocardial hypertrophy (35). Cardiomyocyte-specific knockout of PPAR $\gamma$ in mice results in cardiac hypertrophy (36). Moreover, modulating the activity of PPAR $\gamma$ plays an important role in clinical applications. $\operatorname{PAAR} \gamma$ agonists have been used in clinical as hypoglycemic drugs $(12,37,38)$. In our present work, we demonstrated that PPAR $\gamma$ was downregulated during Ang II-infusion and upregulated by CRP. Importantly, our in vitro and in vivo rescue experiments suggested that PPAR $\gamma$ inhibitor could completely abolish the CRP cardioprotective effects both in NRCMs and in vivo. Therefore, CRP protects against Ang II-induced cardiac hypertrophy and remodeling via activating PPAR $\gamma$.

Cardiac fibrosis is an important hallmark of pathological cardiac hypertrophy and cardiac remodeling $(39,40)$. Therefore, reducing fibroblast accumulation and activation in pathological remodeling is essential for slowing down the fibrosis development as well as improving cardiac function. Here, in our work CRP demonstrated significant antifibrotic effects as evidenced by reduced fibrotic area, decreased collagen expression levels, and reduction of the protein level of $\alpha$-SMA and collagen I. PPAR $\gamma$ activation has been reported to inhibit the pathogenesis of cardiac fibrosis $(41,42)$. Consistent with previous studies, our work showed that PPAR $\gamma$ inhibitor could blunt those antifibrotic effects in Ang II-treated mice accompanied by cardiac dysfunction. Taken together, these data indicated the potential translational implications of CRP in improving diseases with cardiac remodeling.
However, there are still several limitations to this study. Firstly, the specific way of how CRP activates PPAR $\gamma$ and its relationship with PGC- $1 \alpha$ is unclear yet. Secondly, as a typical Chinese herbal medicine, CRP contains a large spectrum of chemical components, such as volatile oils, flavonoids, alkaloids, and so on. Whether one of the components in CRP predominantly acts as the cardioprotective factor or there are cooperative effects among the CRP components are not yet clear. Therefore, further investigations are required to clarify the detailed mechanisms of CRP and its ingredients in preventing pathological cardiac hypertrophy.

\section{Conclusions}

In conclusion, our study demonstrated that CRP could protect against Ang II-induced pathological cardiac hypertrophy by upregulating PPAR $\gamma$. The inhibitory effect of CRP on Ang II-stimulated cardiomyocytes hypertrophy was abolished by PPAR $\gamma$ inhibitor. Moreover, PPAR $\gamma$ inhibitor blocked the protective effects of CRP on cardiac hypertrophy and fibrosis in vivo. Our data presented here provide experimental evidence for the potential application of CRP in the treatment of pathological cardiac hypertrophy. Thus, CRP could be a promising therapeutic prospect for pathological cardiac hypertrophy and heart failure.

\section{Acknowledgments}

Funding: This work was supported by the grants from National Natural Science Foundation of China (81730106 and 81670347 to XL, 81800358 to LW). XL is an Associate Fellow at the Collaborative Innovation Center for Cardiovascular Disease Translational Medicine.

\section{Footnote}

Reporting Checklist: The authors have completed the ARRIVE reporting checklist, available at http://dx.doi. org/10.21037/atm-20-2118

Data Sharing Statement: Available at http://dx.doi. org/10.21037/atm-20-2118

Conflicts of Interest: All authors have completed the ICMJE uniform disclosure form (available at http://dx.doi. org/10.21037/atm-20-2118). XL received research grants 
from Shijiazhuang Yiling Pharmaceutical Co., Ltd. The other authors have no conflicts of interest to declare.

Ethical Statement: The authors are accountable for all aspects of the work in ensuring that questions related to the accuracy or integrity of any part of the work are appropriately investigated and resolved. This study was conducted in accordance to the Guidelines for the Care and Use of Laboratory Animals published by the National Institutes of Health (NIH publication, revised in 2011). All animal experiments were approved by the ethical committees of the Nanjing Medical University (license number: IACUC-1903016).

Open Access Statement: This is an Open Access article distributed in accordance with the Creative Commons Attribution-NonCommercial-NoDerivs 4.0 International License (CC BY-NC-ND 4.0), which permits the noncommercial replication and distribution of the article with the strict proviso that no changes or edits are made and the original work is properly cited (including links to both the formal publication through the relevant DOI and the license). See: https://creativecommons.org/licenses/by-nc-nd/4.0/.

\section{References}

1. Braunwald E. The war against heart failure: the Lancet lecture. Lancet 2015;385:812-24.

2. Cristi-Montero C, Chillon P, Labayen I, et al. Cardiometabolic risk through an integrative classification combining physical activity and sedentary behavior in European adolescents: HELENA study. J Sport Health Sci 2019;8:55-62.

3. Gonzalez A, Ravassa S, Beaumont J, et al. New targets to treat the structural remodeling of the myocardium. J Am Coll Cardiol 2011;58:1833-43.

4. Takano H, Hasegawa H, Nagai T, et al. Implication of cardiac remodeling in heart failure: mechanisms and therapeutic strategies. Intern Med 2003;42:465-9.

5. Nakamura M, Sadoshima J. Mechanisms of physiological and pathological cardiac hypertrophy. Nat Rev Cardiol 2018;15:387-407.

6. Tham YK, Bernardo BC, Ooi JY, et al. Pathophysiology of cardiac hypertrophy and heart failure: signaling pathways and novel therapeutic targets. Arch Toxicol 2015;89:1401-38.

7. Li Y, Liang Y, Zhu Y, et al. Noncoding RNAs in Cardiac Hypertrophy. J Cardiovasc Transl Res 2018;11:439-49.

8. Marian AJ, Braunwald E. Hypertrophic Cardiomyopathy:
Genetics, Pathogenesis, Clinical Manifestations, Diagnosis, and Therapy. Circ Res 2017;121:749-70.

9. Penas FN, Carta D, Cevey AC, et al. Pyridinecarboxylic Acid Derivative Stimulates Pro-Angiogenic Mediators by PI3K/AKT/mTOR and Inhibits Reactive Nitrogen and Oxygen Species and NF-kappaB Activation Through a PPARgamma-Dependent Pathway in T. cruzi-Infected Macrophages. Front Immunol 2020;10:2955.

10. Chen P, Zhan Q, Bai Y, et al. Serum Peroxisome Proliferator-activated Receptor Gamma Coactivator1alpha Related to Myocardial Energy Expenditure in Patients With Chronic Heart Failure. Am J Med Sci 2019;357:205-12.

11. Wang J, Song Y, Zhang Y, et al. Cardiomyocyte overexpression of miR-27b induces cardiac hypertrophy and dysfunction in mice. Cell Res 2012;22:516-27.

12. Cheng HS, Tan WR, Low ZS, et al. Exploration and Development of PPAR Modulators in Health and Disease: An Update of Clinical Evidence. Int J Mol Sci 2019;20.

13. Yu X, Sun S, Guo Y, et al. Citri Reticulatae Pericarpium (Chenpi): Botany, ethnopharmacology, phytochemistry, and pharmacology of a frequently used traditional Chinese medicine. J Ethnopharmacol 2018;220:265-82.

14. Sp N, Kang DY, Joung YH, et al. Nobiletin Inhibits Angiogenesis by Regulating Src/FAK/STAT3-Mediated Signaling through PXN in ER(+) Breast Cancer Cells. Int J Mol Sci 2017;18:935.

15. Moon JY, Cho SK. Nobiletin Induces Protective Autophagy Accompanied by ER-Stress Mediated Apoptosis in Human Gastric Cancer SNU-16 Cells. Molecules 2016;21:914.

16. Zhang X, Zheng K, Li C, et al. Nobiletin inhibits invasion via inhibiting AKT/GSK3beta/beta-catenin signaling pathway in Slug-expressing glioma cells. Oncol Rep 2017;37:2847-56.

17. Chien SY, Hsieh MJ, Chen CJ, et al. Nobiletin inhibits invasion and migration of human nasopharyngeal carcinoma cell lines by involving ERK1/2 and transcriptional inhibition of MMP-2. Expert Opin Ther Targets 2015;19:307-20.

18. Wang F, Chen L, Li FQ, et al. The Increase of Flavonoids in Pericarpium Citri Reticulatae (PCR) Induced by Fungi Promotes the Increase of Antioxidant Activity. Evid Based Complement Alternat Med 2018;2018:2506037.

19. Bentli R, Ciftci O, Cetin A, et al. Oral administration of hesperidin, a citrus flavonone, in rats counteracts the oxidative stress, the inflammatory cytokine production, and the hepatotoxicity induced by the ingestion of 
2,3,7,8-tetrachlorodibenzo-p-dioxin (TCDD). Eur

Cytokine Netw 2013;24:91-6.

20. Lee YS, Cha BY, Choi SS, et al. Nobiletin improves obesity and insulin resistance in high-fat diet-induced obese mice. J Nutr Biochem 2013;24:156-62.

21. Tsutsumi R, Yoshida T, Nii Y, et al. Sudachitin, a polymethoxylated flavone, improves glucose and lipid metabolism by increasing mitochondrial biogenesis in skeletal muscle. Nutr Metab (Lond) 2014;11:32.

22. Ou LJ, Sun XP, Liu QD, et al. Effects of Rhizoma zingiberis and Pericarpium citri reticulatae extracts on myocardial ischemia in rats. Zhong Yao Cai 2009;32:1723-6.

23. Yoshida H, Watanabe H, Ishida A, et al. Naringenin suppresses macrophage infiltration into adipose tissue in an early phase of high-fat diet-induced obesity. Biochem Biophys Res Commun 2014;454:95-101.

24. Yang Z, Liu Y, Deng W, et al. Hesperetin attenuates mitochondria-dependent apoptosis in lipopolysaccharideinduced H9C2 cardiomyocytes. Mol Med Rep 2014;9:1941-6.

25. Shi J, Bei Y, Kong X, et al. miR-17-3p Contributes to Exercise-Induced Cardiac Growth and Protects against Myocardial Ischemia-Reperfusion Injury. Theranostics 2017;7:664-76.

26. Liu Z, Tian H, Hua J, et al. A CRM1 Inhibitor Alleviates Cardiac Hypertrophy and Increases the Nuclear Distribution of NT-PGC-1alpha in NRVMs. Front Pharmacol 2019;10:465.

27. Bhat S, Chin A, Shirakabe A, et al. Recruitment of RNA Polymerase II to Metabolic Gene Promoters Is Inhibited in the Failing Heart Possibly Through PGC-1alpha (Peroxisome Proliferator-Activated Receptor-gamma Coactivator-1alpha) Dysregulation. Circ Heart Fail 2019;12:e005529.

28. Wang H, Bei Y, Lu Y, et al. Exercise Prevents Cardiac Injury and Improves Mitochondrial Biogenesis in Advanced Diabetic Cardiomyopathy with PGC-1alpha and Akt Activation. Cell Physiol Biochem 2015;35:2159-68.

29. Guo J, Tao H, Cao Y, et al. Prevention of Obesity and Type 2 Diabetes with Aged Citrus Peel (Chenpi) Extract. J Agric Food Chem 2016;64:2053-61.

30. Zeng W, Huang KE, Luo Y, et al. Non-targeted urine metabolomics analysis the protective and therapeutic effects of Citri Reticulatae Chachiensis Pericarpium on high-fat feed induced hyperlipidemia in rats. Biomed Chromatogr 2020:e4795.

31. Di W, Lv J, Jiang S, et al. PGC-1: The Energetic Regulator in Cardiac Metabolism. Curr Issues Mol Biol
2018;28:29-46.

32. Zhou H, Li D, Zhu P, et al. Melatonin suppresses platelet activation and function against cardiac ischemia/ reperfusion injury via PPARgamma/FUNDC1/mitophagy pathways. J Pineal Res 2017;63.

33. Ma ZG, Yuan YP, Zhang X, et al. Piperine Attenuates Pathological Cardiac Fibrosis Via PPAR-gamma/AKT Pathways. EBioMedicine 2017;18:179-87.

34. Peng S, Xu J, Ruan W, et al. PPAR-gamma Activation Prevents Septic Cardiac Dysfunction via Inhibition of Apoptosis and Necroptosis. Oxid Med Cell Longev 2017;2017:8326749.

35. Yuan S, Jin J, Chen L, et al. Naoxintong/PPARgamma Signaling Inhibits Cardiac Hypertrophy via Activation of Autophagy. Evid Based Complement Alternat Med 2017;2017:3801976.

36. Duan SZ, Ivashchenko CY, Russell MW, et al. Cardiomyocyte-specific knockout and agonist of peroxisome proliferator-activated receptor-gamma both induce cardiac hypertrophy in mice. Circ Res 2005;97:372-9.

37. Chetty V'T, Sharma AM. Can PPARgamma agonists have a role in the management of obesity-related hypertension? Vascul Pharmacol 2006;45:46-53.

38. Wang W, Zhou X, Kwong JSW, et al. Efficacy and safety of thiazolidinediones in diabetes patients with renal impairment: a systematic review and meta-analysis. Sci Rep 2017;7:1717.

39. Segura AM, Frazier OH, Buja LM. Fibrosis and heart failure. Heart Fail Rev 2014;19:173-85.

40. Kong P, Christia P, Frangogiannis NG. The pathogenesis of cardiac fibrosis. Cell Mol Life Sci 2014;71:549-74.

41. Hou X, Zhang Y, Shen YH, et al. PPAR-gamma activation by rosiglitazone suppresses angiotensin IImediated proliferation and phenotypictransition in cardiac fibroblasts via inhibition of activation of activator protein 1 . Eur J Pharmacol 2013;715:196-203.

42. Yang M, Xiong J, Zou Q, et al. Chrysin attenuates interstitial fibrosis and improves cardiac function in a rat model of acute myocardial infarction. J Mol Histol 2018;49:555-65.

Cite this article as: $\mathrm{Ni} \mathrm{G}$, Wang $\mathrm{K}$, Zhou Y, Wu X, Wang J, Shang H, Wang L, Li X. Citri reticulatae Pericarpium attenuates Ang II-induced pathological cardiac hypertrophy via upregulating peroxisome proliferator-activated receptors gamma. Ann Transl Med 2020;8(17):1064. doi: 10.21037/atm20-2118 